\title{
Acute embolisation of Watchman plug onto aortic bioprosthesis followed by successful percutaneous removal
}

\author{
Jarosław Hiczkiewicz ${ }^{1}$, Konrad Pieszko ${ }^{1}$, Wojciech Faron ${ }^{1}$, Robert Sabiniewicz ${ }^{2}$, Dariusz Hiczkiewicz ${ }^{1}$, \\ Sebastian Łukawiecki ${ }^{1}$ \\ ${ }^{1}$ Department of Cardiology, Multidyscyplinary Public Hospital, Nowa Sol, Poland \\ ${ }^{2}$ Department of Pediatric Cardiology and Congenital Heart Defect, Medical University of Gdansk, Gdansk, Poland
}

Adv Interv Cardiol 2017; 13, 4 (50): 349-350

DOI: https://doi.org/10.5114/aic.2017.71623

Recent years have brought important advances in percutaneous closures of the left atrial appendage (LAA). With increasing experience of operators, the procedure has become a safe and effective alternative to oral anticoagulation in selected patients. Most of the complications regarding this procedure happen in the periprocedural period, and one of the rarest of them is device embolisation, which, as described in the PROTECT_AF study, happened only in 3 of 546 patients (0.6\%) and only in one of them acutely (during the procedure) [1]. In a systematic review, Aminian et al. described 31 cases of device embolisation after percutaneous LAA closure [2]. Most of these complications, however, were acute and almost half of them happened during the procedure. Most of them could also be removed percutaneously, especially if the device embolized into the aorta or left atrial (LA) and not into the LV. Lasek-Bal and Mizia-Stec reported successful closure of the LAA with no significant complications whatsoever [3].

Our patient was an 80-year-old woman who underwent implantation of an aortic bioprosthesis (Shellhigh 27) 8 years earlier due to severe aortic stenosis. She suffered from chronic kidney disease (stage G3a), hypertension and paroxysmal atrial fibrillation, but no coronary artery disease (as it was previously excluded in coronarography). She had a history of multiple severe bleeding from the lower digestive tract during oral anticoagulation with rivaroxaban. After discussing the risks and potential advantages of LAA closure, she was qualified for implantation of the Watchman device.

The procedure initially went with no complications using sedative drugs only and no general anesthesia. Patients' LAA had "chicken wing" morphology and the maximum width of the ostium was $14.1 \mathrm{~mm}$ measured in transesophageal echocardiography (TEE) during the procedure. Initially a Watchman $27 \mathrm{~mm}$ was used, but it had to be switched to a Watchman $21 \mathrm{~mm}$ because of excessive protrusion. Correct localization of the Watchman device was confirmed in fluoroscopy and TEE, as shown in Figures $1 \mathrm{~A}$ and $\mathrm{B}$, respectively, as well as by the tug test with $9 \%$ device compression $(19 / 21 \mathrm{~mm})$. Color Doppler showed no significant peri-device flow. A few minutes after the procedure was finished, while the patient was still in the catheterization laboratory, there was a cardiac arrest with pulseless electrical activity. Immediately we began resuscitation and reintroduced the TEE probe. The echo image showed embolisation of the plug on the aortic bioprosthesis with almost complete obstruction of flow as shown in Figure $1 \mathrm{C}$. During heart massage only a very small jet of flow across the plug was observed as shown in Figure 1 D. Resuscitation was continued according to the European Research Council (ERC) guidelines. Six minutes after arrest ventricular fibrillation (VF) was observed and patient was defibrillated once with $200 \mathrm{~J}$ successfully. Position of the device and lack of sufficient flow through the aortic valve required immediate intervention.

Meanwhile, an EN Snare vascular loop $6 \times 10$ was introduced using a $11 \mathrm{Fr}$ Cordis vascular scaffold in the left femoral artery. The loop was protruded through bioprosthesis valves and we succeeded in catching the device and moving it down to the abdominal aorta. Soon after removing it from the prosthesis, return of spontaneous circulation (ROSC) was observed. Afterwards, using an $18 \times 30$ loop and $16 \mathrm{Fr}$ scaffold, the device was removed completely. Time from embolisation and cardiac arrest to ROSC was about $30 \mathrm{~min}$. We believe that the patient survived only because we had the personnel and equipment prepared for such a situation. The percutaneous device snare and removal was much faster than patient transfer

Corresponding author:

Konrad Pieszko MD, Department of Cardiology, Multidyscyplinary Public Hospital, 7 Chałubińskiego St, 67-100 Nowa Sól, Poland, phone: +487283580 82, e-mail: konrad@pieszko.pl

Received: 6.07.2017, accepted: 16.08.2017. 

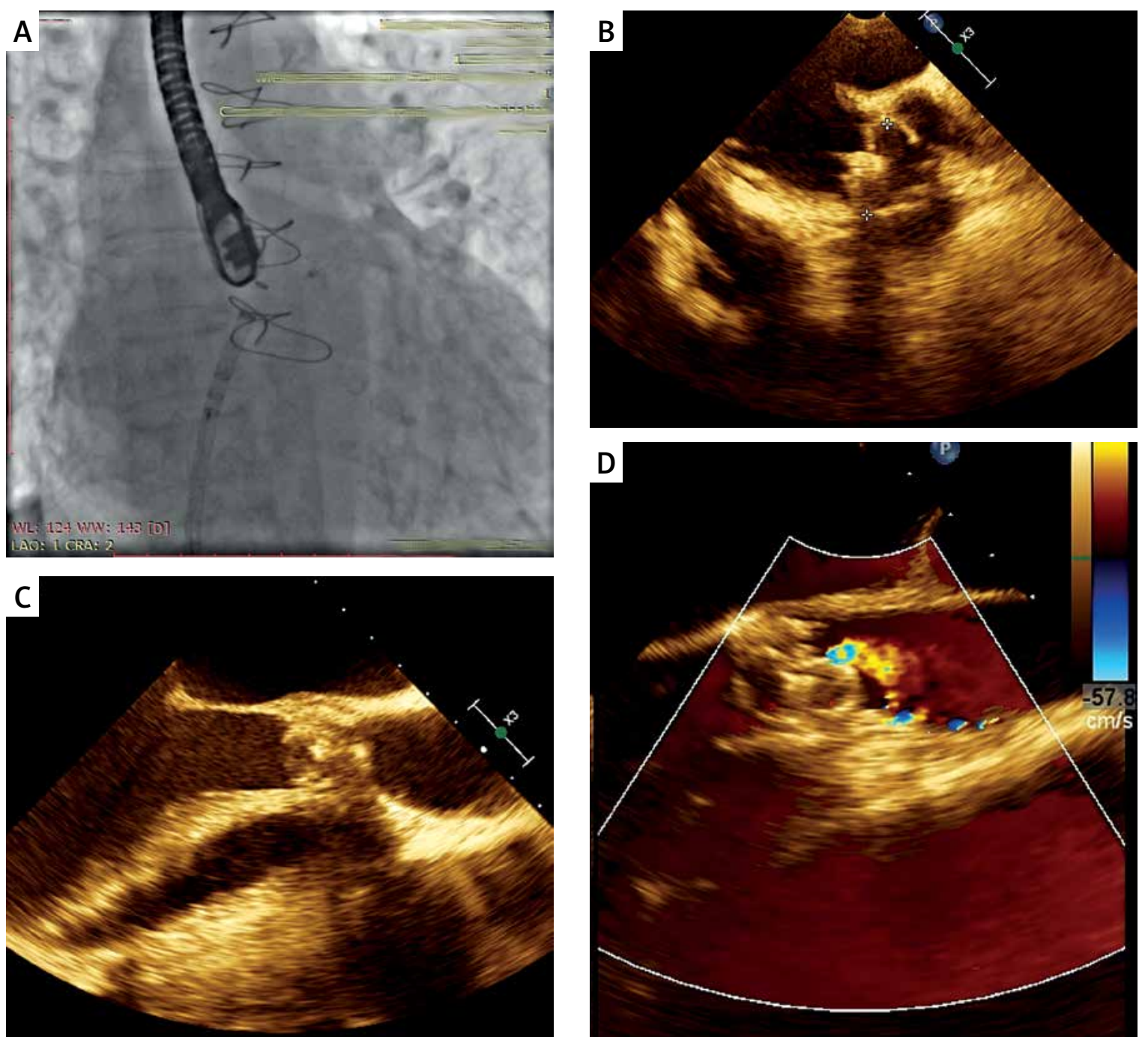

Figure 1. A - Watchmann device placed in LAA, B - confirmation of correct placement in TEE, C - device embolising aortic bioprosthesis, $\mathbf{D}$ - Doppler showing residual flow through the device during reanimation

to the cardiac surgery operating room and surgical removal of the device. In fact, the device was snared during heart massage, which was continued without any interruptions for the whole period of cardiac arrest. This might have prevented neurological losses and contributed to survival.

Afterwards, the patient was placed on the intensive care unit for 24-hour observation. One hour after the procedure the patient had already been extubated and was in full logical contact. One week after the procedure, the patient was discharged with no neurological defects. We suspect that the "chicken wing" morphology might have caused the device to only have a limited area of contact with LAA walls, as can be observed in Figure 1 B. Even though the compression was within the desired range, the outer parts of the device did not expand fully because the volume of the LAA was not proportional to the ostium size. The difficult anatomy of the LAA can increase the risk of device migration. Further studies are needed to evaluate the relation between various LAA morphologies and risk of device embolisation.

\section{Conflict of interest}

The authors declare no conflict of interest.

\section{References}

1. Reddy VY, Holmes D, Doshi SK, et al. Safety of percutaneous left atrial appendage closure: results from the watchman left atrial appendage system for embolic protection in patients with AF (PROTECT AF) clinical trial and the continued access registry. Circulation 2011; 123: 417-24.

2. Aminian A, Lalmand J, Tzikas A, et al. Embolization of left atrial appendage closure devices: a systematic review of cases reported with the Watchman device and the Amplatzer cardiac plug. Catheter Cardiovasc Interv 2015; 86: 128-35.

3. Lasek-Bal A, Mizia-Stec K. Percutaneous closure of the left atrial appendage for secondary prevention of stroke in patients with atrial fibrillation and contraindications to chronic anticoagulant therapy. Postep Kardiol Inter 2015; 11: 14-8. 